\title{
Synchronization in OFDM Based on Piloting Technique
}

\author{
Shweta Kolwal ${ }^{1}$, Sandeep Agrawal ${ }^{2}$ \\ ${ }^{1}$ PG Student, ECE Department, MIT, Ujjain \\ ${ }^{2}$ Assistant Professor, Electronics \& Communication Engineering, MIT Ujjain)
}

\begin{abstract}
In this technical paper A MATLAB SIMULINK MODEL is designed to investigate Pilot Directed Continuous Synchronization of Orthogonal Frequency Division Multiplexing (OFDM) communication systems different mode of channels. This model is valuable for future researchers simulating systems that are too theoretically complex to analyze. The paper details the analysis of a generic OFDM system. An analytical OFDM system using pilot directed synchronization of OFDM with dispersive channel, fading channel and their combination with AWGN channel simulink model used to study Delays between transmitted signal and received signal and Bit synchronization from transmitter to receiver. It also counts the various parameters during communication process like Packet Loss, Bit Loss. This technical paper plays a prominent role in the selection of good quality channel with minimum loss of information with high transmission rate for synchronization of transmitter with receiver.
\end{abstract}

Keywords: OFDM, ICI, ISI, MIMO, Chu symbol

\section{Introduction}

The evolution of OFDM can be divided into three parts. There are consists of Frequency Division Multiplexing (FDM), Multicarrier Communication (MC) and Orthogonal Frequency Division Multiplexing. The objective of this model is to develop and explore a simulink model of Pilot Directed Continuous Synchronization of OFDM (Using 64QAM rate $2 / 3,2 \mathrm{~K}$ and $1 / 32$ guard interval). Synchronization is basically used for error free reception of incoming data without delay. The pilot tones are used to estimate the channel magnitude and phase. These estimates have now have gone from flat lines (a perfect zero delay channel). As we know that by increasing Orthogonality the data rate is decreases because of synchronization problem. When we used 16 QAM modulation the rate of transmission is low as compared to 64 QAM modulation technique but it have one drawback that its Orthogonality is less as compared to 64 QAM modulation scheme. Modulation technique with different combination of channels (Dispersive, AWGN+ Dispersive, Fading and Fading+ AWGN channel) between transmitter and receiver and to study Packet Loss, Bit Loss and effect on bit rate.

\subsection{Orthogonality}

Two signals are orthogonal if their dot product is zero. That is, if you take two signals multiply them together and if their integral over an interval is zero, then two signals are orthogonal in that interval. Mathematically, suppose we have a set of signals $\Psi$ then,

$$
\int_{a}^{a+T} \Psi_{p}(t) \Psi_{q}^{*}(t)=\left\{\begin{array}{l}
K \text { for } p=q \\
0 \text { for } p \neq q
\end{array}\right.
$$

The signals are orthogonal if the integral value is zero over the interval $[\mathrm{a}, \mathrm{a}+\mathrm{T}]$, where $\mathrm{T}$ is the symbol period. This way of transmitting information helps in handling the effects of multipath propagation efficiently. The transmission is efficient because of the spectral overlap of narrowband subcarriers. OFDM supports various modulations techniques like BPSK, QAM, and QPSK etc. QAM is further divided in 16, 32 and 64 QAM techniques. In a classical parallel data system, the total signal frequency band is divided into $\mathrm{N}$ no overlapping frequency sub channels. Each sub channel is modulated with a separate symbol and then the $\mathrm{N}$ sub channels are frequency-multiplexed.

\subsection{General block diagram of OFDM System} 1.3

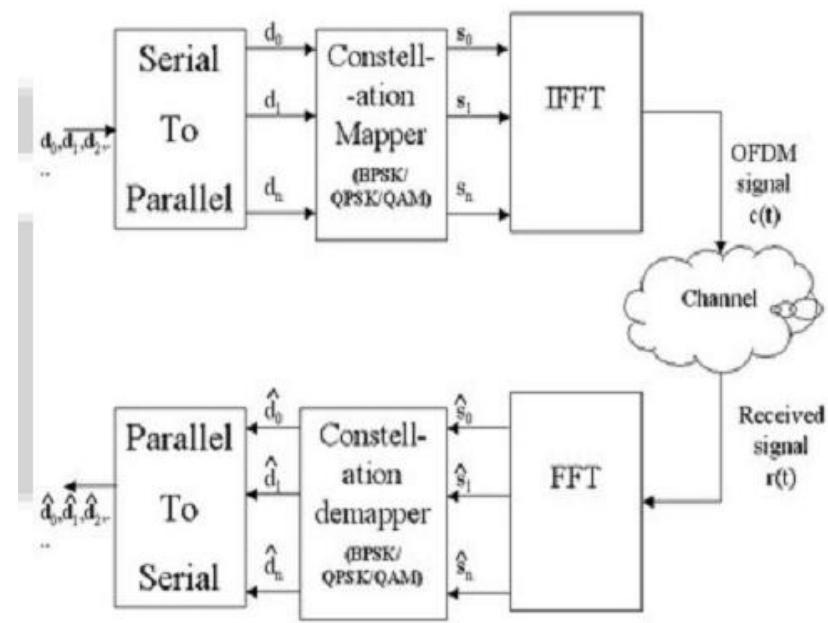

Figure 1: Block diagram of OFDM

An OFDM System consist mainly three block elements transmitter, receiver and channel. Bandwidth: Occupied bandwidth is of course directly related to the data rate to transmit. However, the question is, what is the minimum bandwidth to take in order to obtain enough diversity and avoid the loss off all the signal in frequency selective fading environments. On the other hand much bandwidth means also much transmitting power. There is a tradeoff between bandwidth and transmitted power. That optimal bandwidth is found by channel simulations and field test trials. In DAB, for example, a bandwidth of $1,5 \mathrm{MHz}$ is a good compromise for the type of propagation conditions that apply. 


\section{International Journal of Science and Research (IJSR) \\ ISSN (Online): 2319-7064}

Index Copernicus Value (2015): 78.96 | Impact Factor (2015): 6.391

Number of carriers: We have seen that the greater the number of carriers, the greater the symbol period on each carrier and so lees equalization is needed and the greater the diversity offered by the system. However, with differential modulation, it is important that the channel not vary too much during one symbol period. This is not the case when the receiver is moving because of Doppler Effect and short term fading. Then a great number of carriers will limit the moving speed. This is another trade off of OFDM. Another problem is the complexity in the implementation increase when carrier number increases because large FFT are needed. To continue with the DAB example, 1536 carriers has been found to be a good compromise. That led to a carrier spacing of $1 \mathrm{kHz}$ and a symbol period of $1 \mathrm{~ms}$. Moving speed of mobiles shouldn't get over $160 \mathrm{~km}$ per hour.

Guard interval: The trade-off of guard interval is to set it large enough to avoid inter-symbol interference depending on the memory of channel and transmitter position spacing in a single frequency network. On the other hand, we want it to be as small as possible as it carries no information and can be seen as a spoil of bandwidth. In wireless systems, a guard interval of $25 \%$ of symbol period is often met and seems to be a good compromise. That is the value taken for DAB; it allows a maximum distance of about 80 Kilometres between transmitters. At the reception, it is very important to distinguish the starting point of FFT to avoid wrong demodulation. And so synchronization has to be precise. It explains the use of special symbols (pilot) for synchronization in transmission. Hardware design of transmitter and receiver is important because of high peak to average ratio which causes distortions if dynamic range of amplifier and converters is not high enough. OFDM is very sensitive to carrier frequency offsets. Such offsets are mainly the cause of receiver local oscillator's instability and Doppler Effect when mobile is moving.

\section{Pilot Directed Continuous Synchronization of OFDM Simulink Model}

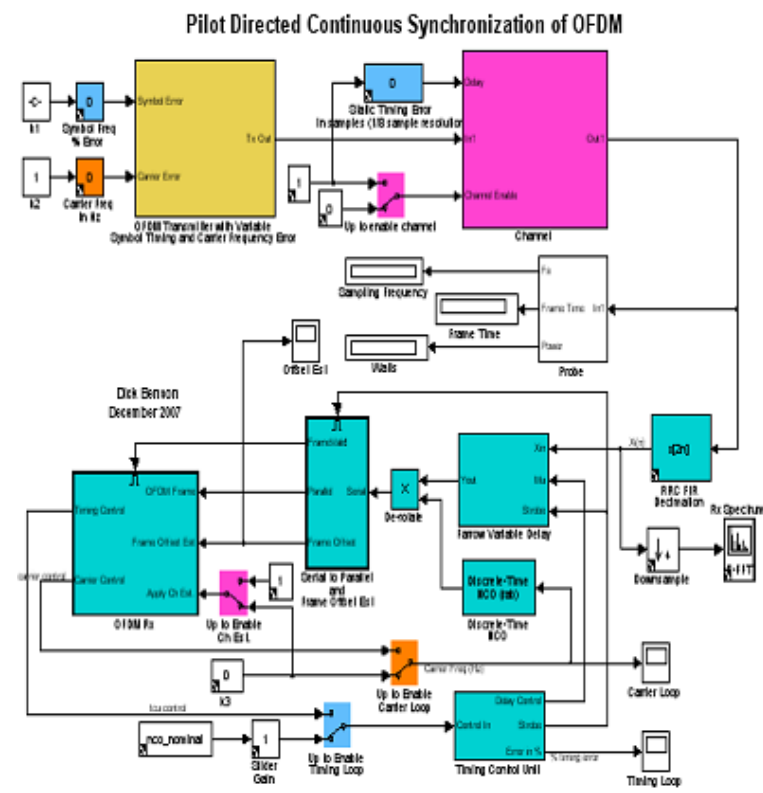

Figure 2: Pilot directed continuous synchronization of
Magenta: channel related colour,

Orange: carrier frequency selected switch,

Light Blue: symbol timing (both symbol rate and static time offset). Running the model with these settings (no impairments) represents the perfect world situation which is never realized in practice but serves as a starting point.

Magenta: this slide is used for the channel the on position is upper side and off position is down side.

Orange: carrier frequency related the on position is upper side and off position is down side while the Light blue is used for symbol timing. Notice the four sliders have 0 values. There are 6 selections of channel impairments selected by the magenta "Channel Select" slider:

$0=$ none

$1=$ dispersive

$2=$ fading

$3=\mathrm{AWGN}$

$4=$ dispersive + AWGN

$5=$ fading + AWGN

The RX and pilot tone constellations are ideal:
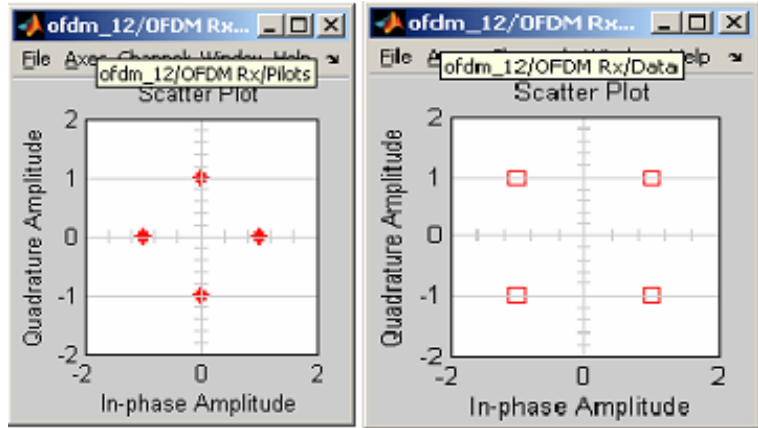

Figure 2 (a): ideal response of pilot tones and RX .

2) Next, enable the channel impairment by changing the MAGENTA slider to a ONE. This inserts a multi- tap complex FIR filter (dispersive channel) into the path between TX and RX. The RX data and pilot tone constellations are now:

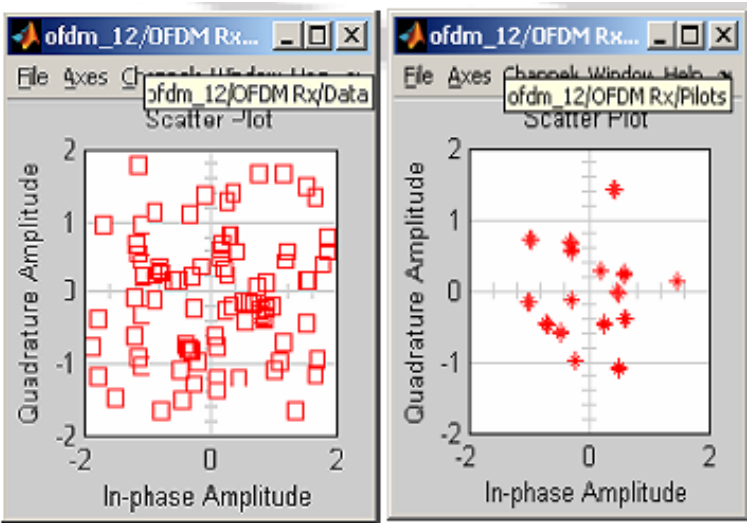

Figure 2 (b): Dispersive channel

As you can see, the RX data is hopelessly scrambled by the effects of the channel. The pilot tones are used to estimate the channel magnitude and phase. These estimates have now have gone from flat lines (a perfect zero delay channel) to: 


\section{International Journal of Science and Research (IJSR) \\ ISSN (Online): 2319-7064}

Index Copernicus Value (2015): 78.96 | Impact Factor (2015): 6.391

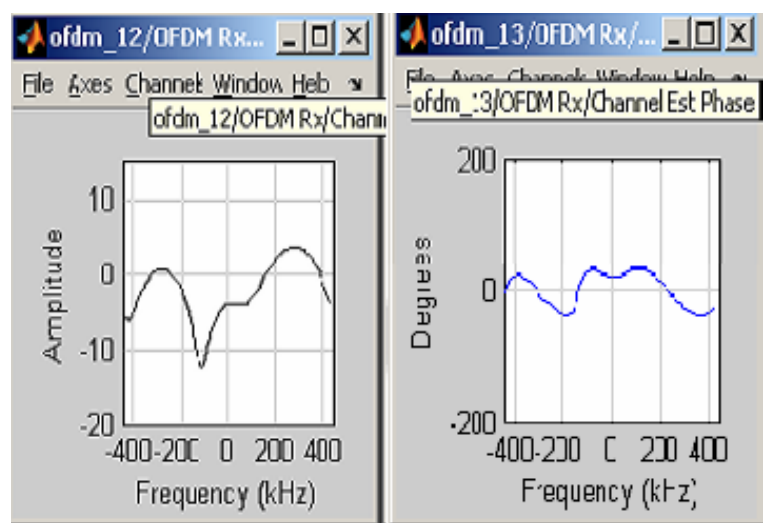

Figure 2 (c)

Next, incorporate these channel estimations in the RX by changing the MAGENTA switch in the RX to the UP position (Up to Enable Channel Estimation). The RX data constellation should now look like this:

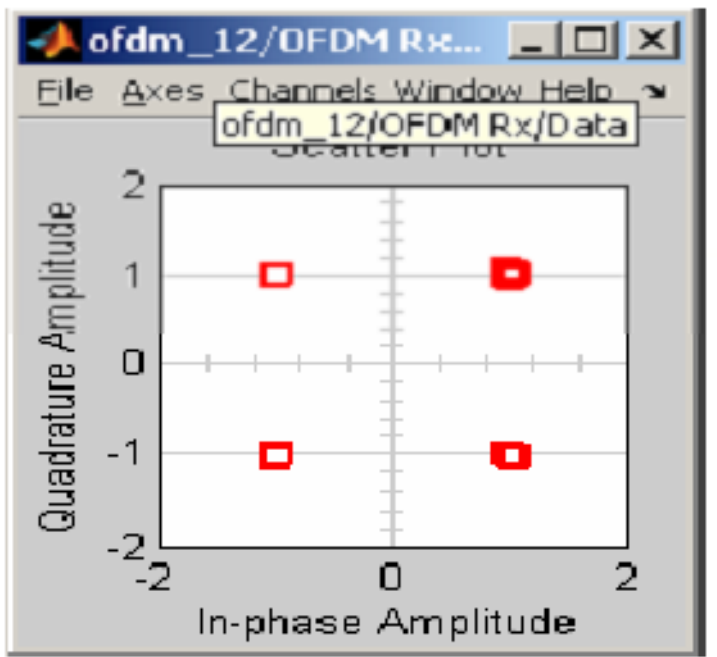

Figure 2 (d): Graph for ON position of magenta switch.

While the pilot constellation will be unchanged. The above plot indicates that the errors that were being introduced by the channel are being successfully mitigated.

3) Next, introduce a TX carrier frequency error of $550 \mathrm{~Hz}$ by changing the ORANGE slider outside the TX subsystem from 0 to 600

The RX data and pilot constellations will looks as follows:

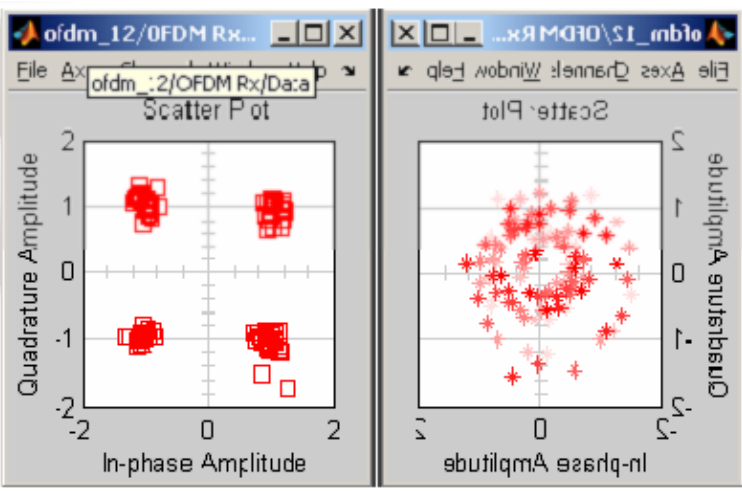

Figure 2 (e): RX and Pilot Tones
Note the pilots are not static, but are rotating about the centre. The phase estimate of channel is helping the RX data quite a bit. But the correction is NOT perfect because there is a time varying phase (watch the channel estimation phase plot) that is not constant over a symbol period. The higher the carrier offset frequency, the more the phase changes over one OFDM symbol time, and the more difficult the situation. To fix this, activate the carrier loop in the RX by changing the ORANGE switch (Up to Enable Carrier Loop) to the up position. The RX and pilot constellations should now look as follows:

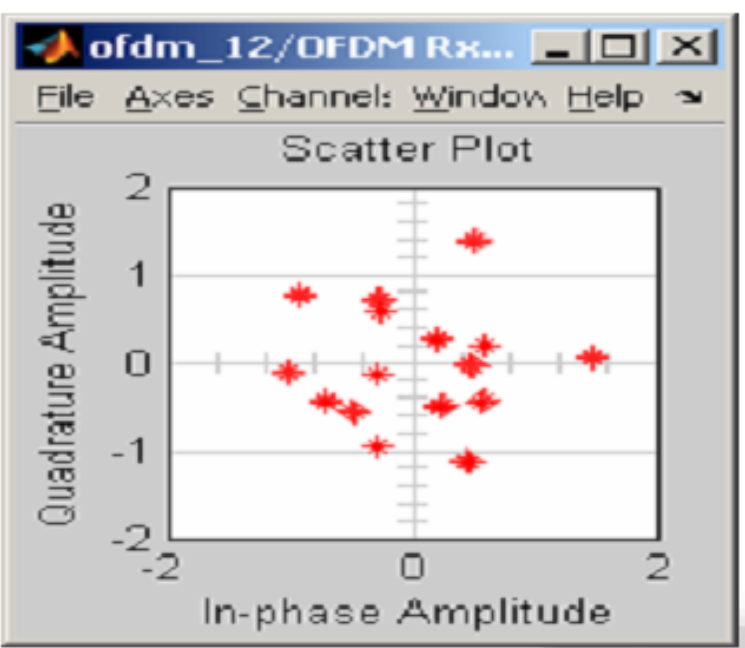

Figure 2 (f): After activation carrier loop switch.

Note that the pilots are no longer rotating and the channel phase estimate is stable as well. With this switch up, the Carrier Loop control is now driving a complex NCO to "derotate" the OFDM signal and doing (virtually) all of the work to correct for this impairment.

4) The next and most difficult impairment to correct is symbol rate error. Introduce $+0.2 \% \mathrm{TX}$ symbol frequency error (LIGHT BLUE slider in TX) and notice the RX and Pilot constellations are again hopelessly received the "FrameOffset in Samples" numeric display. It will be decreasing in value as the simulation proceeds. This represents a COARSE estimation of how far the TX frame has drifted (due to its symbol frequency error) with respect to the expected RX frame timing. To mitigate this, while the simulation is running, change the LIGHT BLUE switch in the RX ("Up to Enable Timing Loop") to the UP position. Let the simulation run and you will see that the numeric display will be driven back to approximately zero. This coarse estimate of the time offset is used to steer the RX timing to a point where it is close enough to activate the FINE TIMING control loop. When this is done (it is automatic), you will see that the RX data and pilot constellations are now being properly recovered. 


\section{International Journal of Science and Research (IJSR) \\ ISSN (Online): 2319-7064}

Index Copernicus Value (2015): 78.96 | Impact Factor (2015): 6.391

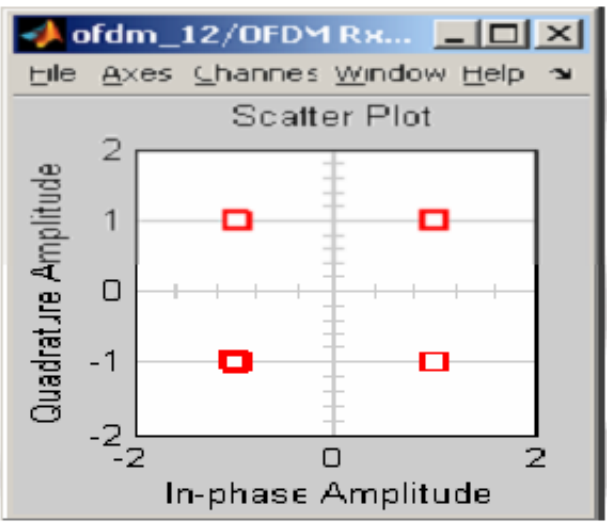

Figure 2 (g)

Finally, introduce a step change in the TX timing by changing the LIGHT BLUE Static Timing Error channel slider from 0 to a positive value of circa 10 samples. This will cause the RX to come out of fine timing lock and the coarse timing estimator will then be used to reacquire OFDM synch. This is the sort of channel timing change one might experience when crossing from one cell site into another or is simply the norm when the RX starts up. Changes in the latest model include:

1) The channel model has been enhanced to include a variety of channel impairments that can be selected by changing the slider. Note that the slider should be set to an integer value to be certain of the selection being made.

2) A BER indicator. To compare BER results, set a simulation run time (e.g. 0.03 seconds), start the simulation, and then note the number of errors after the simulation stops.

3) Loop tuning to improve RX robustness with a fading channel.

\section{Limitations}

1) Synchronization: One of the crucial problems in the receiver is to sample the incoming signal correctly. If the wrong sequence of samples is processed, the Fast Fourier Transform shall not correctly recover the received data on the carriers.

2) Orthogonality: We know that several carriers are actually advantageous whenever they are mathematically orthogonal. So carriers Orthogonality is a constrain that can leads to a wrong operation of OFDM systems if not respected. The Orthogonality is provided by IFFT

\section{Applications}

For developing different applications we need some kind of sequence:

1) The channel model has been enhanced to include a variety of channel impairments that can be selected by changing the slider. Note that the slider should be set to an integer value to be certain of the selection being made.

2) A BER indicator. To compare BER results, set a simulation run time (e.g. 0.03 seconds), start the simulation, and then note the number of errors after the simulation stops. On the basis of above information this model is very useful for 3G, Digital Video Broadcasting, High speed LAN network and in HDSL.

\section{Future Improvements}

Pilot Directed OFDM Using 64 QAM Synchronization model is very useful in digital video broadcasting and in Multi Carrier CDMA (MC-CDMA).Since the transmission rate high so it can also useful in forthcoming 4G technology. HDSL: High bit rate Digital Subscriber Line.

\section{Conclusion}

At the time of reception, it is very important to distinguish the starting point of FFT to avoid wrong demodulation. And so synchronization has to be precise. It explains the use of special symbols (pilot) for synchronization in transmission. Hardware design of Transmitter and receiver is important because of high peak to average ratio which causes distortions if dynamic range of amplifiers and converters is not high enough. OFDM is very sensitive to carrier frequency offsets. Such offsets are mainly the cause of receiver local oscillators' instability and Doppler Effect when mobile is moving.

\section{References}

[1] S.Weinstein and P.Ebert,'Data Transmission by Frequency Division Multiplexing Using the Discrete Fourier Transform" IEEE Trans. On Communication, vol.19, Issue: 5, pp. 628-634, Oct.1971

[2] A.Peled and A. Ruiz, "Frequency domain data transmission using reduced computational complexity algorithms, Acoustics, Speech, and Signal Processing" IEEE International Conference on ICASSP '80, vol. 5, pp.964-967, Apr. 1980.

[3] Senior Capstone Project 2007 Final Report by: Jarrod Cook Nathan Gove Project Advisors: Dr. Brian Huggins Dr. In Soo Ahn Dr. Prasad Shastry.

[4] F.H. Raab, "Efficiency of Doherty RF power-amplifier systems," IEEE Trans. Broadcast., vol.BC-33, no.3, pp.77-83, 1987.

[5] Takenaka, H. Takahashi, K. Ishikura, K. Hasegawa, K. Asano, and M. Kanamori, "A 240W Doherty GaAspower FET amplifier with high efficiency and low distortion for W-CDMA base stations, " MWE 2004 Workshop Digest, WS12-1, pp.299-304, 2004.

[6] Efficacy of Multiband OFDM Approach in High Data Rate Ultra WideBand WPAN Physical Layer Standard using Realistic Channel Models Bikramaditya Das Department of Electrical Engineering National Institute of Technology, Rourkela -769008, India Susmita Das, Member, IEEE Department of Electrical Engineering National Institute of Technology, Rourkela -769008, India

[7] S. Roy, J. R. Foerster, V. S. Somayazulu and D.G. Leeper, "Ultrawideband radio design: the promise of high- speed, shortrange wireless connectivity, Proceedings of the IEEE, Vol. 92, Issue 2, pp. 295-311, 2004.

[8] Rajbir and Vikash "Simulink Model of OFDM Using 64Qam with Different Combination of Channels “. 3-537

\section{Volume 6 Issue 7, July 2017 www.ijsr.net}

